\title{
(Anti)chiral Superfield Approach to Nilpotent Symmetries: Self-Dual Chiral Bosonic Theory
}

\author{
N. Srinivas, ${ }^{1}$ T. Bhanja, ${ }^{1}$ and R. P. Malik ${ }^{1,2}$ \\ ${ }^{1}$ Physics Department, Centre of Advanced Studies, Banaras Hindu University, Varanasi 221 005, India \\ ${ }^{2}$ DST Centre for Interdisciplinary Mathematical Sciences, Faculty of Science, Banaras Hindu University, Varanasi 221 005, India \\ Correspondence should be addressed to T. Bhanja; tapobroto.bhanja@gmail.com
}

Received 21 November 2016; Accepted 29 January 2017; Published 7 March 2017

Academic Editor: George Siopsis

Copyright (c) 2017 N. Srinivas et al. This is an open access article distributed under the Creative Commons Attribution License, which permits unrestricted use, distribution, and reproduction in any medium, provided the original work is properly cited. The publication of this article was funded by $\mathrm{SCOAP}^{3}$.

\begin{abstract}
We exploit the beauty and strength of the symmetry invariant restrictions on the (anti)chiral superfields to derive the BecchiRouet-Stora-Tyutin (BRST), anti-BRST, and (anti-)co-BRST symmetry transformations in the case of a two $(1+1)$-dimensional $(2 D)$ self-dual chiral bosonic field theory within the framework of augmented (anti)chiral superfield formalism. Our $2 D$ ordinary theory is generalized onto a $(2,2)$-dimensional supermanifold which is parameterized by the superspace variable $Z^{M}=\left(x^{\mu}, \theta, \bar{\theta}\right)$, where $x^{\mu}$ (with $\mu=0,1$ ) are the ordinary $2 D$ bosonic coordinates and $(\theta, \bar{\theta}$ ) are a pair of Grassmannian variables with their standard relationships: $\theta^{2}=\bar{\theta}^{2}=0, \theta \bar{\theta}+\bar{\theta} \theta=0$. We impose the (anti-)BRST and (anti-)co-BRST invariant restrictions on the (anti)chiral superfields (defined on the (anti)chiral $(2,1)$-dimensional supersubmanifolds of the above general $(2,2)$-dimensional supermanifold) to derive the above nilpotent symmetries. We do not exploit the mathematical strength of the (dual-)horizontality conditions anywhere in our present investigation. We also discuss the properties of nilpotency, absolute anticommutativity, and (anti-)BRST and (anti-)co-BRST symmetry invariance of the Lagrangian density within the framework of our augmented (anti)chiral superfield formalism. Our observation of the absolute anticommutativity property is a completely novel result in view of the fact that we have considered only the (anti)chiral superfields in our present endeavor.
\end{abstract}

\section{Introduction}

The model of the $2 D$ self-dual chiral bosonic field theory has found applications in different areas of research in theoretical physics, for example, models of (super)strings, W-gravities, quantum Hall effect, and $2 D$ statistical systems (see, e.g., [112] for details). This model has been shown to provide a physical example of Hodge theory [13]. The purpose of our present investigation is to apply the augmented version of (anti)chiral superfield approach to BRST formalism [14-17] to discuss this $2 D$ system in a systematic manner.

The usual superfield approach [18-24] to Becchi-RouetStora-Tyutin (BRST) formalism takes into account the mathematical strength and beauty of the horizontality condition (HC) to obtain the off-shell nilpotent and absolutely anticommuting (anti-)BRST symmetry transformations for the gauge and associated (anti-)ghost fields of a given $p$-form $(p=1,2,3, \ldots)$ gauge theory. This has been systematically generalized so as to derive the proper (anti-)BRST symmetry transformations $\left(s_{(a) b}\right)$ for the matter, gauge and (anti-)ghost fields together for a given interacting $p$-form gauge theory (see, e.g., [25-28]). The latter superfield formalism exploits the additional restrictions (e.g., gauge invariant restrictions) which are found to be consistent with the celebrated HC. These theoretical formulations have been christened as the augmented version of superfield formalism (see, e.g., [2528]).

In our present investigation, we apply the augmented version of the (anti)chiral superfield formalism to the model of $2 D$ self-dual chiral bosonic field theory where we exploit the theoretical power and potential of the symmetry invariant restrictions on the (anti)chiral superfields to derive the proper (anti-)BRST and (anti-)co-BRST symmetry transformations. We do not exploit the mathematical technique of 
(dual-)HCs anywhere in our present endeavor. This is a novel feature in our present theoretical method (which thoroughly relies on the beauty and strength of the physically motivated symmetry considerations). Such methods have also been applied in the context of $\mathcal{N}=2$ SUSY quantum mechanical models [29-32] where the nilpotent $\mathscr{N}=2$ SUSY symmetry transformations have been derived. From now onwards, we shall be calling our present (anti)chiral superfield approach as the "augmented superfield formalism" for the sake of brevity but we shall always use the (anti)chiral superfields for our further discussions.

To be specific, we wish to mention that, within the framework of the usual superfield approach to BRST formalism, a given $D$-dimensional ordinary $p$-form gauge theory is generalized onto a $(D, 2)$-dimensional supermanifold which is characterized by the superspace coordinates $Z^{M}=\left(x^{\mu}, \theta, \bar{\theta}\right)$, where $x^{\mu}$ (with $\mu=0,1,2, \ldots, D-1$ ) correspond to the bosonic coordinates and a pair of Grassmannian variables $(\theta, \bar{\theta})$ satisfy $\theta^{2}=\bar{\theta}^{2}=0, \theta \bar{\theta}+\bar{\theta} \theta=0$. The ordinary fields of the gauge theory (which are function of spacetime coordinates $x^{\mu}$ and defined on an ordinary Minkowskian spacetime manifold) are generalized to the superfields that are function of the superspace coordinates $Z^{M}$ and defined on the $(D, 2)$ dimensional supermanifold. The HC requires that the super curvature $(p+1)$-form should be equated with the ordinary curvature $(p+1)$-form. This process of covariant reduction leads to the derivation of the proper (i.e., off-shell nilpotent and absolutely anticommuting) (anti-)BRST symmetry transformations. It turns out that these symmetry transformations (and their corresponding generators) are identified with the translational generators $\left(\partial_{\theta}, \partial_{\bar{\theta}}\right)$ along the Grassmannian directions of the $(D, 2)$-dimensional supermanifold.

Against the backdrop of the above explanations, it is clear that the nilpotency $\left(\partial_{\theta}^{2}=\partial_{\bar{\theta}}^{2}=0\right)$ and the absolute anticommutativity $\left(\partial_{\theta} \partial_{\bar{\theta}}+\partial_{\bar{\theta}} \partial_{\theta}=0\right)$ of the translational generators $\left(\partial_{\theta}, \partial_{\bar{\theta}}\right)$ entail upon the (anti-)BRST symmetry transformations $\left(s_{(a) b}\right)$ and their corresponding generators $\left(Q_{(a) b}\right)$ to obey the properties of nilpotency $\left(s_{(a) b}^{2}=0, Q_{(a) b}^{2}=\right.$ $0)$ and the absolute anticommutativity $\left(s_{b} s_{a b}+s_{a b} s_{b}=0\right.$, $\left.Q_{b} Q_{a b}+Q_{a b} Q_{b}=0\right)$. This has been found to be true in the works by Bonora and Tonin [20, 21] where the superfields have been expanded along all the Grassmannian directions $(1, \theta, \bar{\theta}, \theta \bar{\theta})$ of the $(D, 2)$-dimensional supermanifold. As a consequence, the properties of the nilpotency and absolute anticommutativity of the (anti-)BRST symmetry transformations (and their corresponding generators) follow. In our present endeavor, for the sake of simplicity, we do not take the full expansion of the superfields along all the Grassmannian directions. As a consequence, our present (anti)chiral superfield approach to BRST formalism is simple.

One of the key features of our present method is the use of (anti)chiral superfields that are defined on the (anti)chiral supersubmanifolds of the general $(2,2)$-dimensional supermanifold on which our $2 \mathrm{D}$ self-dual chiral bosonic theory is generalized. We capture the nilpotency and absolute anticommutativity properties of the (anti-)BRST and (anti-)co-BRST symmetries and their generators in our present formalism. One of the novel results of our present endeavor is the observation that the absolute anticommutativity property of the (anti-)BRST as well as (anti-)co-BRST charges emerges very naturally in our present investigation despite the fact that we have taken only the (anti)chiral super expansions of the (anti)chiral superfields that are defined on the (anti)chiral supersubmanifolds.

Our present endeavor is essential on the following grounds. First and foremost, the symmetry invariant restrictions have played a decisive role in the derivation of the BRSTtype symmetries in the context of gauge theories (see, e.g., $[33,34])$ as well as $\mathcal{N}=2$ SUSY symmetries of the quantum mechanical models [29-32]. We have recently applied this method of derivation in the context of $4 D$ Abelian 2-form gauge theory [33] as well as a $1 D$ toy model of a rigid rotor [34]. Thus, it is important for us to see its sanctity in the context of $2 D$ model of the self-dual chiral bosonic theory which has different dimensionality compared to $4 D$ and $1 D$. We have accomplished this goal in our present endeavor. Second, our method of derivation is simple and physically intuitive where we have not used the (dual-)horizontality conditions that are mathematical in nature. Finally, our present endeavor is an attempt to provide theoretical richness in the context of the application of superfield formalism to derive the BRST-type nilpotent symmetries for the $p$-form gauge theories (in any arbitrary dimension of spacetime).

Our present paper is organized as follows. First of all, to set up the notations and convention, we recapitulate the bare essentials of the (anti-)BRST and (anti-)co-BRST symmetries of our theory in Section 2 within the framework of Lagrangian formulation. Section 3 deals with the derivation of (anti-)BRST transformations by exploiting the (anti-)BRST invariant restrictions on the (anti)chiral superfields. The subject matter of Section 4 is connected with the derivation of (anti-)co-BRST symmetries from the appropriate restrictions on the (anti)chiral superfields. Section 5 is devoted to the discussion of nilpotent (anti-)BRST and (anti-)co-BRST invariance of the Lagrangian density within the framework of augmented superfield formalism. In Section 6, we discuss the properties of nilpotency and absolute anticommutativity of the (anti-)BRST and (anti-)co-BRST transformations and corresponding charges. We finally make some concluding remarks in Section 7.

\section{Preliminaries: Lagrangian Formulation}

We begin with the following (anti-)BRST and (anti-)coBRST invariant Lagrangian density for the $2 D$ self-dual chiral bosonic field theory (see, e.g., [35] for details):

$$
\begin{aligned}
\mathscr{L}_{b}= & \frac{\dot{\phi}^{2}}{2}-\frac{\dot{v}^{2}}{2}+\dot{v}\left(v^{\prime}-\phi^{\prime}\right)+\lambda\left[\dot{\phi}-\dot{v}+v^{\prime}-\phi^{\prime}\right] \\
& -\frac{1}{2}\left(\phi^{\prime}-v^{\prime}\right)^{2}+B(\dot{\lambda}-v-\phi)+\frac{B^{2}}{2}-i \dot{\bar{C}} \dot{C} \\
& +2 i \bar{C} C,
\end{aligned}
$$

where the overdot on fields $\phi$ and $v$ (i.e., $\dot{\phi}=\partial \phi / \partial t, \dot{v}=\partial v / \partial t$ ) denotes the derivative w.r.t. the "time" evolution parameter $t$ and the prime on the fields (i.e., $\phi^{\prime}$ and $v^{\prime}$ ) stands for the space 
derivatives (i.e., $\phi^{\prime}=\partial \phi / \partial x, v^{\prime}=\partial v / \partial x$ ). This establishes the fact that $\phi$ and $v$ are function of the spacetime variables $(x$ and $t$ ). The dynamics of the $2 D$ self-dual chiral bosonic field theory are such that the evolution parameter $t$ plays a decisive as well as very special role (see, e.g., [35] for details). In the above, we have $\lambda$ as the "gauge" field and $B$ is the NakanishiLautrup type auxiliary field. The fermionic $\left(C^{2}=\bar{C}^{2}=0\right.$, $C \bar{C}+\bar{C} C=0$ ) (anti-)ghost fields $(\bar{C}) C$ are required for the sake of validity of unitarity in the theory. The gauge-fixing term in the theory is the following: $-(1 / 2)(\dot{\lambda}-v-\phi)^{2} \equiv B(\dot{\lambda}-$ $v-\phi)+B^{2} / 2$.

It is straightforward to check the following nilpotent $\left(s_{(a) b}^{2}=0\right)\left(\right.$ anti-)BRST symmetry transformations $s_{(a) b}$ (see, e.g., $[13,35])$ :

$$
\begin{aligned}
s_{a b} \phi & =-\bar{C}, \\
s_{a b} v & =-\bar{C}, \\
s_{a b} \lambda & =\dot{\bar{C}}, \\
s_{a b} \bar{C} & =0, \\
s_{a b} C & =-i B, \\
s_{a b} B & =0, \\
s_{b} \phi & =-C, \\
s_{b} v & =-C, \\
s_{b} \lambda & =\dot{C}, \\
s_{b} \bar{C} & =+i B, \\
s_{b} C & =0, \\
s_{b} B & =0 ;
\end{aligned}
$$

leave the action integral $S=\int d^{2} x \mathscr{L}_{b} \equiv \int d x \int d t \mathscr{L}_{b}$ invariant because the Lagrangian density $\mathscr{L}_{b}$ transforms to the total "time" derivatives as follows:

$$
\begin{aligned}
s_{a b} \mathscr{L}_{b} & =\frac{\partial}{\partial t}[B \dot{\bar{C}}], \\
s_{b} \mathscr{L}_{b} & =\frac{\partial}{\partial t}[B \dot{C}] .
\end{aligned}
$$

Exploiting the standard tricks and techniques of the Noether theorem, we note that the above infinitesimal, continuous, and nilpotent $\left(s_{(a) b}^{2}=0\right)$ and absolutely anticommuting $\left(s_{b} s_{a b}+s_{a b} s_{b}=0\right)$ (anti-)BRST symmetry transformations $\left(s_{(a) b}\right)$ lead to the derivation of nilpotent $\left(Q_{(a) b}^{2}=0\right)$ and absolutely anticommuting $\left(Q_{b} Q_{a b}+Q_{a b} Q_{b}=0\right)$ conserved charges $Q_{(a) b}$ as follows:

$$
\begin{aligned}
Q_{a b} & =\int d x\left[B \dot{\bar{C}}-\left(\dot{\phi}-\dot{v}+v^{\prime}-\phi^{\prime}\right) \bar{C}\right] \\
& \equiv \int d x[B \dot{\bar{C}}-\dot{B} \bar{C}]
\end{aligned}
$$

$$
\begin{aligned}
Q_{b} & =\int d x\left[B \dot{C}-\left(\dot{\phi}-\dot{v}+v^{\prime}-\phi^{\prime}\right) C\right] \\
& \equiv \int d x[B \dot{C}-\dot{B} C] .
\end{aligned}
$$

The conservation law of the above (anti-)BRST charges $\left(Q_{(a) b}\right)$ can be proven by exploiting the following EulerLagrange (EL) equations of motion (EOM):

$$
\begin{aligned}
\dot{B} & =\dot{\phi}-\dot{v}+v^{\prime}-\phi^{\prime}, \\
B & =v+\phi-\dot{\lambda}, \\
\ddot{C}+2 C & =0, \\
-B & =\ddot{\phi}+\dot{\lambda}-\dot{v}^{\prime}-\lambda^{\prime}-\left(\phi^{\prime \prime}-v^{\prime \prime}\right), \\
\ddot{\bar{C}}+2 \bar{C} & =0, \\
B & =\ddot{v}-2 \dot{v}^{\prime}+\dot{\phi}^{\prime}+\dot{\lambda}-\lambda^{\prime}-\left(\phi^{\prime \prime}-v^{\prime \prime}\right),
\end{aligned}
$$

that emerge from the Lagrangian density $\mathscr{L}_{b}$ [cf. (1)]. We note that $(-2 B)=\ddot{\phi}-\ddot{v}+\dot{v}^{\prime}-\dot{\phi}^{\prime} \equiv \ddot{B}$ from (5) which implies that $\ddot{B}+2 B=0$ is an off-shoot of the above EL-EOMs which plays an important role in the proof of conservation law (i.e., $\left.\dot{Q}_{(a) b}=0\right)$.

Beside the above continuous nilpotent symmetry transformations (2), we have another set of nilpotent symmetry transformations in the theory. These (anti-)dual-BRST [i.e., (anti-)co-BRST] symmetry transformations $\left(s_{(a) d}\right)$ are (see, e.g. [13]) as follows:

$$
\begin{aligned}
s_{a d} \lambda & =C, \\
s_{a d} \phi & =\frac{1}{2} \dot{C}, \\
s_{a d} v & =\frac{1}{2} \dot{C}, \\
s_{a d} C & =0, \\
s_{a d} \bar{C} & =\frac{i}{2}\left(\dot{\phi}-\dot{v}+v^{\prime}-\phi^{\prime}\right), \\
s_{a d}\left(\dot{\phi}-\dot{v}+v^{\prime}-\phi^{\prime}\right) & =0, \\
s_{a d} B & =0, \\
s_{d} \lambda & =\bar{C}, \\
s_{d} \phi & =\frac{1}{2} \dot{\bar{C}}, \\
s_{d} v & =\frac{1}{2} \dot{\bar{C}}, \\
s_{d} \bar{C} & =0, \\
s_{d} C & =-\frac{i}{2}\left(\dot{\phi}-\dot{v}+v^{\prime}-\phi^{\prime}\right),
\end{aligned}
$$




$$
\begin{aligned}
s_{d}\left(\dot{\phi}-\dot{v}+v^{\prime}-\phi^{\prime}\right) & =0, \\
s_{d} B & =0 .
\end{aligned}
$$

It can be readily checked that the Lagrangian density transforms to the total "time" derivatives under the above (anti-)co-BRST symmetry transformations, namely;

$$
\begin{gathered}
s_{a d} \mathscr{L}_{b}=\frac{\partial}{\partial t}\left[\frac{\dot{C}}{2}\left(\dot{\phi}-\dot{v}+v^{\prime}-\phi^{\prime}\right)\right], \\
s_{d} \mathscr{L}_{b}=\frac{\partial}{\partial t}\left[\frac{\dot{\bar{C}}}{2}\left(\dot{\phi}-\dot{v}+v^{\prime}-\phi^{\prime}\right)\right] .
\end{gathered}
$$

As a consequence, the action integral $S=\int d x \int d t \mathscr{L}_{b}$ remains invariant under the (anti-)co-BRST symmetry transformations, too. Exploiting the basic tenets of Noether theorem, we obtain the following conserved (anti-)co-BRST [i.e., (anti-)dual-BRST] charges:

$$
\begin{aligned}
Q_{a d}=\int d x\left[\frac{\dot{C}}{2}\left(\dot{\phi}-\dot{v}+v^{\prime}-\phi^{\prime}\right)-C(\dot{\lambda}-v-\phi)\right] \\
\equiv \int d x\left[\frac{\dot{B} \dot{C}}{2}+B C\right] \equiv \frac{1}{2} \int d x[\dot{B} \dot{C}-\ddot{B} C], \\
\quad(B=-(\dot{\lambda}-v-\phi), \ddot{B}+2 B=0), \\
Q_{d}=\int d x\left[\frac{\dot{\bar{C}}}{2}\left(\dot{\phi}-\dot{v}+v^{\prime}-\phi^{\prime}\right)-\bar{C}(\dot{\lambda}-v-\phi)\right] \\
\equiv \int d x\left[\frac{\dot{B} \overline{\bar{C}}}{2}+B \bar{C}\right] \equiv \frac{1}{2} \int d x[\dot{B} \dot{\bar{C}}-\ddot{B} \bar{C}], \\
\quad(B=-(\dot{\lambda}-v-\phi), \ddot{B}+2 B=0) .
\end{aligned}
$$

The conservation law (i.e., $\dot{Q}_{(a) d}=0$ ) of these charges can be proven by exploiting the EL-EOMs in (5) which are derived from the Lagrangian density (1). We would like to lay emphasis on the fact that one of the key signatures of the (anti-)co-BRST symmetry transformations $\left(s_{(a) d}\right)$ is the observation that the gauge-fixing term (i.e., $\left.-(1 / 2)(\dot{\lambda}-v-\phi)^{2}\right)$ remains invariant (i.e., $\left.s_{(a) d}[\dot{\lambda}-v-\phi]=0\right)$ under them.

In the forthcoming sections, we shall capture all the theoretical ingredients (i.e., symmetries, conserved charges, (anti-)BRST, and (anti-)co-BRST invariances), discussed in this section, within the framework of augmented version of the (anti)chiral superfield formalism and provide geometrical meaning to them in the language of specific operators defined on the (anti)chiral supersubmanifolds.

\section{3. (Anti-)BRST Symmetries: Superfield Approach}

To derive the off-shell nilpotent (anti-)BRST symmetry $s_{(a) b}$ within the framework of superfield formalism, first of all, we generalize the $2 D$ fields $(\phi(x), v(x), \lambda(x), C(x), \bar{C}(x))$ onto the $(2,1)$-dimensional (anti)chiral supermanifolds which are characterized by the superspace coordinates $\left(x^{\mu}, \bar{\theta}\right)$ and $\left(x^{\mu}, \theta\right)$, respectively. For instance, for the derivation of the BRST symmetry transformations $\left(s_{b}\right)$, we generalize the $2 D$ ordinary fields to their counterpart antichiral superfields on a $(2,1)$-dimensional antichiral supersubmanifold as

$$
\begin{aligned}
& \phi(x) \longrightarrow \widetilde{\Phi}(x, \bar{\theta})=\phi(x)+i \bar{\theta} f_{1}(x), \\
& v(x) \longrightarrow \widetilde{V}(x, \bar{\theta})=v(x)+i \bar{\theta} f_{2}(x) \\
& \lambda(x) \longrightarrow \Lambda(x, \bar{\theta})=\lambda(x)+\bar{\theta} R(x), \\
& C(x) \longrightarrow F(x, \bar{\theta})=C(x)+i \bar{\theta} b_{1}(x) \\
& \bar{C}(x) \longrightarrow \bar{F}(x, \bar{\theta})=\bar{C}(x)+i \bar{\theta} b_{2}(x)
\end{aligned}
$$

where the sets of fields $\left(f_{1}(x), f_{2}(x), R(x), b_{1}(x), b_{2}(x)\right)$, on the right hand side, are the secondary fields which are to be determined in terms of the basic and auxiliary fields of the Lagrangian density (1) of our theory. We note that the generalization and super expansion of the NakanishiLautrup type of auxiliary field $B(x)$ have not been taken into account because they remain invariant under the (anti-)BRST and (anti-)co-BRST symmetry transformations (i.e., $s_{(a) b} B=$ $\left.s_{(a) d} B=0\right)$. In other words, there are no (anti)chiral expansions for this field which implies that the superfield generalization is $B(x) \rightarrow \widetilde{B}^{(\mathscr{B}, A B, D, A D)}(x, \bar{\theta})=B(x)$. Here we have taken the results of earlier works [20, 21, 25-28] where it has been established that the coefficients of $\theta$ and/or $\bar{\theta}$ correspond to the nilpotent symmetries.

In this context, it is pertinent to point out that the BRST invariant quantities (when generalized onto a $(2,1)$-dimensional antichiral supersubmanifold) must be independent of the "soul" coordinate $\bar{\theta}$ because the latter is not physically realized. This is one of the key and basic tenets of the augmented antichiral superfield formalism. It is gratifying to state that the following BRST invariant quantities,

$$
\begin{aligned}
s_{b} C & =0, \\
s_{b}(\phi-v) & =0, \\
s_{b}(\lambda \dot{C}) & =0, \\
s_{b}(\lambda+\dot{\phi}) & =0, \\
s_{b}[\dot{B} \lambda+\dot{i} \bar{C}] & =0, \\
s_{b}(\phi C) & =0, \\
s_{b}[B(\phi+v)-2 i \bar{C} C] & =0,
\end{aligned}
$$

turn out to be useful for us in the BRST invariant restrictions on the antichiral superfields of the $(2,1)$-dimensional antichiral supersubmanifold. 
Against the backdrop of the above arguments, it is interesting to note that we have the following restrictions on the antichiral (super)fields of our theory, namely;

$$
\begin{aligned}
& \widetilde{\Phi}(x, \bar{\theta})-\widetilde{V}(x, \bar{\theta})=\phi(x)-v(x), \\
& F^{(\mathscr{B})}(x, \bar{\theta})=C(x), \\
& \widetilde{\Phi}(x, \bar{\theta}) F^{(\mathscr{B})}(x, \bar{\theta})=\phi(x) C(x), \\
& B(x)[\widetilde{\Phi}(x, \bar{\theta})+\widetilde{V}(x, \bar{\theta})]-2 i \bar{F}(x, \bar{\theta}) F^{(\mathscr{B})}(x, \bar{\theta}) \\
& \quad=B(x)[\phi(x)+v(x)]-2 i \bar{C}(x) C(x), \\
& \dot{B}(x) \Lambda(x, \bar{\theta})+i \dot{\bar{F}}(x, \bar{\theta}) \dot{F}^{(\mathscr{B})}(x, \bar{\theta}) \\
& \quad=\dot{B}(x) \lambda(x)+\dot{\bar{C}}(x) \dot{C}(x), \\
& \Lambda(x, \bar{\theta}) \dot{F}^{(\mathscr{B})}(x, \bar{\theta})=\lambda(x) \dot{C}(x), \\
& \Lambda(x, \bar{\theta})+\dot{\widetilde{\Phi}}(x, \bar{\theta})=\lambda(x)+\dot{\phi}(x),
\end{aligned}
$$

where $F^{(\mathscr{B})}(x, \bar{\theta})=C(x)$ because $s_{b} C=0$. As a consequence of the BRST invariance $s_{b} C=0$, there is no expansion of the superfield $F(x, \bar{\theta})$ along $\bar{\theta}$-direction (i.e., $\left.b_{1}(x)=0\right)$. The superscript $(\mathscr{B})$ denotes that the superfield $F(x, \bar{\theta})$ has been derived after the application of the BRST invariant restriction (i.e., $s_{b} C=0$ ). In addition to this restriction, we have the following relationships that emerge from the above BRST invariant restrictions, namely;

$$
\begin{aligned}
f_{1}(x) & =f_{2}(x)=f(x), \\
b_{1}(x) & =0, \\
R(x) \dot{C}(x) & =0, \\
R(x)+i \dot{f}(x) & =0, \\
\dot{B}(x) R(x)-\dot{b}_{2}(x) \dot{C}(x) & =0, \\
i B(x) f(x)+b_{2}(x) C(x) & =0, \\
f(x) C(x) & =0 .
\end{aligned}
$$

The last entry shows that $f(x)$ is proportional to $C(x)$. For the sake of algebraic convenience, we choose the nontrivial solution as $f(x)=i C(x)$. This choice, immediately, entails upon the other secondary fields to be $R(x)=\dot{C}(x), b_{2}(x)=B(x)$. Thus, we have the following expressions for the secondary fields in terms of the basic and auxiliary fields:

$$
\begin{aligned}
& R(x)=\dot{C}(x), \\
& b_{2}(x)=B(x), \\
& f(x)=i C(x), \\
& b_{1}(x)=0 .
\end{aligned}
$$

We lay emphasis on the fact that our choice may differ by an overall constant factor. As a consequence, the expressions in (13) would also change accordingly in a consistent manner. We note that this kind of freedom is also present in our original continuous symmetry transformations (2). The substitution of these values in the antichiral expansions (9) leads to the following:

$$
\begin{aligned}
\widetilde{\Phi}^{(\mathscr{B})}(x, \bar{\theta}) & =\phi(x)+\bar{\theta}(-C(x)) \\
& \equiv \phi(x)+\bar{\theta}\left(s_{b} \phi(x)\right), \\
\widetilde{V}^{(\mathscr{B})}(x, \bar{\theta}) & =v(x)+\bar{\theta}(-C(x)) \equiv v(x)+\bar{\theta}\left(s_{b} v(x)\right), \\
\Lambda^{(\mathscr{B})}(x, \bar{\theta}) & =\lambda(x)+\bar{\theta}(\dot{C}(x)) \equiv \lambda(x)+\bar{\theta}\left(s_{b} \lambda(x)\right), \\
F^{(\mathscr{B})}(x, \bar{\theta}) & =C(x)+\bar{\theta}(0) \equiv C(x)+\bar{\theta}\left(s_{b} C(x)\right), \\
\bar{F}^{(\mathscr{B})}(x, \bar{\theta}) & =\bar{C}(x)+\bar{\theta}(i B) \equiv \bar{C}(x)+\bar{\theta}\left(s_{b} \bar{C}(x)\right),
\end{aligned}
$$

where the superscript $(\mathscr{B})$ stands for the expansion of superfields after the application of BRST invariant restrictions (11). We note that the coefficient of $\bar{\theta}$ is nothing but the BRST symmetry transformations [cf. (2)] in the above expansions.

We now concentrate on the derivation of the anti-BRST symmetry transformations from our augmented superfield formalism. Towards this goal in mind, first of all, we generalize the ordinary $2 D$ fields of the theory onto the superfields defined on the chiral supersubmanifold that is characterized by the superspace coordinates $\left(x^{\mu}, \theta\right)$. Thus, we have the following generalizations and expansions of the chiral superfields, namely;

$$
\begin{aligned}
& \phi(x) \longrightarrow \widetilde{\Phi}(x, \theta)=\phi(x)+i \theta \bar{f}_{1}(x), \\
& v(x) \longrightarrow \widetilde{V}(x, \theta)=v(x)+i \theta \bar{f}_{2}(x), \\
& \lambda(x) \longrightarrow \Lambda(x, \theta)=\lambda(x)+\theta \bar{R}(x), \\
& C(x) \longrightarrow F(x, \theta)=C(x)+i \theta \bar{b}_{1}(x), \\
& \bar{C}(x) \longrightarrow \bar{F}(x, \theta)=\bar{C}(x)+i \theta \bar{b}_{2}(x),
\end{aligned}
$$

where on the r.h.s., we have the secondary fields $\left(\bar{f}_{1}(x)\right.$, $\left.\bar{f}_{2}(x), \bar{R}(x), \bar{b}_{1}(x), \bar{b}_{2}(x)\right)$ which are to be determined by exploiting the anti-BRST restrictions. In this context, we note that the following set of anti-BRST invariant quantities,

$$
\begin{aligned}
s_{a b}(\bar{C}) & =0, \\
s_{a b}(\phi-v) & =0, \\
s_{a b}(\lambda \dot{\bar{C}}) & =0, \\
s_{a b}(\lambda+\dot{\phi}) & =0, \\
s_{a b}[\dot{B} \lambda+i \dot{\bar{C}} \dot{C}] & =0, \\
s_{a b}(\phi C) & =0, \\
s_{a b}[B(\phi+v)-2 i \bar{C} C] & =0,
\end{aligned}
$$


provides us with useful relationships that can be generalized onto the $(2,1)$-dimensional chiral supersubmanifold (of the general $(2,2)$-dimensional supermanifold).

Against the backdrop of the above logic, we have the following restrictions on the chiral (super)fields defined on the $(2,1)$-dimensional chiral supersubmanifold:

$$
\begin{aligned}
& \widetilde{\Phi}(x, \theta)-\widetilde{V}(x, \theta)=\phi(x)-v(x), \\
& \bar{F}^{(A B)}(x, \theta)=\bar{C}(x) \\
& \Lambda(x, \theta) \dot{\bar{F}}(x, \theta)=\lambda(x) \dot{\bar{C}}(x), \\
& \Lambda(x, \theta)+\dot{\widetilde{\Phi}}(x, \theta)=\lambda(x)+\dot{\phi}(x) \\
& \widetilde{\Phi}(x, \theta) \bar{F}^{(A B)}(x, \theta)=\phi(x) \bar{C}(x), \\
& B(x)(\widetilde{\Phi}(x, \theta)+\widetilde{V}(x, \theta))-2 i \bar{F}^{(A B)}(x, \theta) F(x, \theta) \\
& \quad=B(x)(\phi(x)+v(x))-2 i \bar{C}(x) C(x), \\
& \dot{B}(x) \Lambda(x, \theta)+i \dot{\bar{F}}^{(A B)}(x, \theta) \dot{F}(x, \theta) \\
& \quad=\dot{B}(x) \lambda(x)+i \dot{\bar{C}}^{(x)} \dot{C}(x)
\end{aligned}
$$

where we have taken $\bar{F}^{(A B)}(x, \theta)=\bar{C}(x)$ due to the anti-BRST invariance $\left(s_{a b} \bar{C}=0\right)$. The above anti-BRST restrictions lead to the following useful relationships amongst the secondary fields and the basic and auxiliary fields of our theory (cf. (1)), namely;

$$
\begin{aligned}
\bar{f}_{1}(x) & =\bar{f}_{2}(x)=\bar{f}(x), \\
\bar{b}_{2}(x) & =0, \\
\bar{R}(x)+\dot{\overline{i f}}(x) & =0, \\
\bar{f}(x) \bar{C}(x) & =0, \\
\bar{R}(x) \dot{\bar{C}}(x) & =0, \\
\dot{B}(x) \bar{R}(x)+\dot{\bar{b}}_{1}(x) \dot{\bar{C}}(x) & =0, \\
i \bar{f} B(x)-\bar{b}_{1} \bar{C}(x) & =0 .
\end{aligned}
$$

We have the freedom to choose the expression for $\bar{R}(x)$ in terms of $\dot{\bar{C}}(x)$ which can differ by an overall constant factor. Accordingly, other expressions for secondary fields would change in (18). However, this freedom is also present in the original transformations (2) where we have the freedom to multiply the transformations by a constant overall factor (without changing the symmetry property of our theory). A nontrivial and simple solution for $\bar{R}(x)$ is $\bar{R}(x)=\dot{\bar{C}}(x)$. This choice, immediately, implies the following:

$$
\begin{aligned}
& \bar{b}_{1}(x)=-B(x), \\
& \bar{f}(x)=i \bar{C}(x), \\
& \bar{b}_{2}(x)=0 .
\end{aligned}
$$

Plugging these values of the secondary fields into (15) yields

$$
\begin{aligned}
\widetilde{\Phi}^{(A B)}(x, \theta) & =\phi(x)+\theta(-\bar{C}(x)) \\
& \equiv \phi(x)+\theta\left(s_{a b} \phi(x)\right), \\
\widetilde{V}^{(A B)}(x, \theta) & =v(x)+\theta(-\bar{C}(x)) \\
& \equiv v(x)+\theta\left(s_{a b} v(x)\right), \\
\Lambda^{(A B)}(x, \theta) & =\lambda(x)+\theta(\dot{\bar{C}}(x)) \\
& \equiv \lambda(x)+\theta\left(s_{a b} \lambda(x)\right), \\
F^{(A B)}(x, \theta) & =C(x)+\theta(-i B(x)) \\
& \equiv C(x)+\theta\left(s_{a b} C(x)\right), \\
\bar{F}^{(A B)}(x, \theta) & =\bar{C}(x)+\theta(0) \equiv \bar{C}(x)+\theta\left(s_{a b} \bar{C}(x)\right),
\end{aligned}
$$

where the superscript $(A B)$ on the superfields denotes that these have been obtained after the application of the antiBRST invariant restrictions (17). Thus, the coefficient of $\theta$, in the above expansions, is nothing but the anti-BRST symmetry transformations (2).

A close look at (14) and (20) implies that there is an intimate relationship between the continuous (anti-)BRST symmetry transformations (as well as their generators) and the translational generators $\left(\partial_{\theta}, \partial_{\bar{\theta}}\right)$ on the (anti)chiral supersubmanifolds of the general supermanifold. In other words, we have the following relationships:

$$
\begin{gathered}
\frac{\partial}{\partial \theta} \Omega^{(A B)}(x, \theta)=s_{a b} \omega(x) \equiv \pm i\left[w(x), Q_{a b}\right]_{ \pm}, \\
\frac{\partial}{\partial \bar{\theta}} \Omega^{(\mathscr{B})}(x, \bar{\theta})=s_{b} \omega(x) \equiv \pm i\left[w(x), Q_{b}\right]_{ \pm},
\end{gathered}
$$

where the $( \pm)$ signs, as the subscripts on the square bracket, denote the (anti)commutator for the generic fields $\omega(x)$ being (fermionic) bosonic in nature. The above equations demonstrate that the operators $\left(s_{b}, \partial_{\bar{\theta}}, Q_{b}\right)$ and $\left(s_{a b}, \partial_{\theta}, Q_{a b}\right)$ are interrelated. Their nilpotency property (i.e., $s_{(a) b}^{2}=0, \partial_{\theta}^{2}=0, \partial_{\bar{\theta}}^{2}=$ $\left.0, Q_{(a) b}^{2}=0\right)$ is intertwined and there is a geometrical interpretation for the (anti-)BRST symmetry transformations. For instance, the relationship in (21) states that the BRST transformations on the generic field $\omega(x)$ in the $2 D$ ordinary space are equivalent to the translation of the corresponding superfield $\Omega^{(\mathscr{B})}(x, \bar{\theta})$ (obtained after exploiting the BRST invariant restrictions [cf. (17)]) along the Grassmannian direction $\bar{\theta}$ of the $(2,1)$-dimensional antichiral supersubmanifold (of the general $(2,2)$-dimensional supermanifold). In exactly similar fashion, the geometrical interpretation for the nilpotent $\left(s_{a b}^{2}=0\right)$ anti-BRST symmetry transformations $\left(s_{a b}\right)$ can be provided. 


\section{4. (Anti-)co-BRST Symmetries: Superfield Formalism}

First of all, we concentrate on the derivation of dual-BRST (i.e., co-BRST) symmetry transformations of our theory by exploiting the antichiral superfields whose expansions, along the $\bar{\theta}$-direction of the $(2,1)$-dimensional antichiral supersubmanifold, are given in (9). Towards this goal in mind, we note that the following useful and interesting set of co-BRST (or dual-BRST) invariant quantities, namely;

$$
\begin{aligned}
s_{d}(\phi-v) & =0, \\
s_{d}(\bar{C}) & =0, \\
s_{d}(\lambda \bar{C}) & =0, \\
s_{d}(\dot{\lambda}-2 \phi) & =0, \\
s_{d}(\phi C) & =0, \\
s_{d}\left[\phi\left(\dot{\phi}-\dot{v}+v^{\prime}-\phi^{\prime}\right)+i \dot{\bar{C}} C\right] & =0, \\
s_{d}\left[\lambda\left(\dot{\phi}-\dot{v}+v^{\prime}-\phi^{\prime}\right)+2 i \bar{C} C\right] & =0,
\end{aligned}
$$

can be generalized onto the $(2,1)$-dimensional antichiral supersubmanifold and the following co-BRST invariant restrictions can be imposed on the antichiral superfields:

$$
\begin{aligned}
& \widetilde{\Phi}(x, \bar{\theta})-\widetilde{V}(x, \bar{\theta})=\phi(x)-v(x), \\
& \bar{F}^{(D)}(x, \bar{\theta})=\bar{C}(x), \\
& \Lambda(x, \bar{\theta}) \bar{F}^{(D)}(x, \bar{\theta})=\lambda(x) \bar{C}(x), \\
& \widetilde{\Phi}(x, \bar{\theta}) \dot{\bar{F}}^{(D)}(x, \bar{\theta})=\phi(x) \dot{\bar{C}}(x), \\
& \dot{\Lambda}(x, \bar{\theta})-2 \widetilde{\Phi}(x, \bar{\theta})=\dot{\lambda}(x)-2 \phi(x), \\
& \Lambda(x, \bar{\theta})\left[\dot{\widetilde{\Phi}}(x, \bar{\theta})-\dot{\widetilde{V}}(x, \bar{\theta})+\widetilde{V}^{\prime}(x, \bar{\theta})-\widetilde{\Phi}^{\prime}(x, \bar{\theta})\right] \\
& \quad+2 i \bar{F}^{(D)}(x, \bar{\theta}) F(x, \bar{\theta})=\lambda(x) \\
& \quad \cdot\left[\dot{\phi}(x)-\dot{v}(x)+v^{\prime}(x)-\phi^{\prime}(x)\right]+2 i \bar{C}(x) C(x), \\
& \widetilde{\Phi}(x, \bar{\theta})\left[\dot{\tilde{\Phi}}(x, \bar{\theta})-\dot{\widetilde{V}}(x, \bar{\theta})+\widetilde{V}^{\prime}(x, \bar{\theta})-\widetilde{\Phi}^{\prime}(x, \bar{\theta})\right] \\
& \quad+\dot{i \bar{F}}(D)(x, \bar{\theta}) F(x, \bar{\theta})=\phi(x) \\
& \quad \cdot\left[\dot{\phi}(x)-\dot{v}(x)+v^{\prime}(x)-\phi^{\prime}(x)\right]+\dot{\bar{C}}(x) C(x) .
\end{aligned}
$$

The above co-BRST invariant restrictions imply that the l.h.s. should, primarily, remain independent of the $\bar{\theta}$-coordinate. In other words, we demand that the co-BRST invariant quantities (which are physical in some sense) should remain independent of the "soul" coordinates. The aforementioned are not physical objects because they are only mathematical artifacts which are used in the superspace formalism. It is worthwhile to mention here that, in the older literature (see, e.g., [36]), the Grassmannian variables $\theta$ and $\bar{\theta}$ have been christened as the "soul" coordinates and the bosonic variables $x^{\mu}(\mu=0,1,2, \ldots, D-1)$ have been called as the "body" coordinates. In the above (23), we have taken $\bar{F}^{(D)}(x, \bar{\theta})=\bar{C}(x)$ because the anti-ghost field $\bar{C}(x)$ remains invariant $\left(s_{d} \bar{C}(x)=\right.$ $0)$ under $s_{d}$. Here we have taken the results from the earlier works $[20,21]$ where it has been shown that the coefficients of $\theta$ and $\bar{\theta}$ in this expansion of superfields (along the Grassmannian directions of the appropriately chosen supermanifold) lead to the derivation of nilpotent symmetries.

The co-BRST invariant restrictions (23) lead to the following relationships between the secondary fields and basic fields:

$$
\begin{aligned}
& f_{1}(x)=f_{2}(x)=f(x), \\
& R(x) \bar{C}(x)=0, \\
& f(x) \dot{\bar{C}}(x)=0, \\
& R(x)\left[\dot{\phi}(x)+\dot{v}(x)+v^{\prime}(x)-\phi^{\prime}(x)\right]+2 \bar{C}(x) b_{1}(x) \\
& \quad=0 \\
& \dot{R}(x)-2 i f(x)=0, \\
& \text { if }(x)\left[\dot{\phi}(x)-\dot{v}(x)+v^{\prime}(x)-\phi^{\prime}(x)\right]+\dot{\bar{C}}(x) b_{1}(x) \\
& \quad=0 .
\end{aligned}
$$

It is clear that the nontrivial solutions of the restrictions $R(x) \bar{C}(x)=0$ and $f(x) \bar{C}(x)=0$ are $R(x) \propto \bar{C}(x)$ and $f(x) \propto \dot{\bar{C}}(x)$. However, if we make one of the simplest choices, say $R(x)=\bar{C}(x)$, the following relationships automatically ensue:

$$
\begin{aligned}
& f(x)=-\frac{i}{2} \dot{\bar{C}}(x), \\
& b_{1}(x)=-\frac{1}{2}\left[\dot{\phi}(x)-\dot{v}(x)+v^{\prime}(x)-\phi^{\prime}(x)\right], \\
& b_{2}(x)=0 .
\end{aligned}
$$

The substitution of these values of the secondary fields into expansions (9) leads to the following expansions (in terms of the co-BRST transformations $s_{d}$ ):

$$
\begin{aligned}
& \widetilde{\Phi}^{(D)}(x, \bar{\theta})=\phi(x)+\bar{\theta}\left(\frac{\dot{\bar{C}}(x)}{2}\right) \\
& \equiv \phi(x)+\bar{\theta}\left(s_{d} \phi(x)\right), \\
& \widetilde{V}^{(D)}(x, \bar{\theta})=v(x)+\bar{\theta}\left(\frac{\dot{\bar{C}}(x)}{2}\right) \\
& \equiv v(x)+\bar{\theta}\left(s_{d} v(x)\right), \\
& \Lambda^{(D)}(x, \bar{\theta})=\lambda(x)+\bar{\theta}(\bar{C}(x)) \equiv \lambda(x)+\bar{\theta}\left(s_{d} \lambda(x)\right),
\end{aligned}
$$




$$
\begin{aligned}
& F^{(D)}(x, \bar{\theta}) \\
& =C(x)+\bar{\theta}\left[-\frac{i}{2}\left\{\dot{\phi}(x)-\dot{v}(x)+v^{\prime}(x)-\phi^{\prime}(x)\right\}\right] \\
& \quad \equiv C(x)+\bar{\theta}\left(s_{d} C(x)\right) \\
& \bar{F}^{(D)}(x, \bar{\theta})=\bar{C}(x)+\bar{\theta}(0) \equiv \bar{C}(x)+\bar{\theta}\left(s_{d} \bar{C}(x)\right) .
\end{aligned}
$$

The superscript $(D)$ on the superfields in (23) and (26) denotes that the above expansions have been obtained after the application of the co-BRST (or dual-BRST) invariant restrictions (23). It is clear that the coefficients of $\bar{\theta}$, on the r.h.s., yield the proper (i.e., off-shell nilpotent) dual-BRST symmetry transformations.

To derive the anti-co-BRST symmetry transformation $\left(s_{a d}\right)$, we tap the chiral expansion (15) and impose the antico-BRST invariant restrictions on these superfields. In this context, it can be readily checked that we have the validity of the following:

$$
\begin{aligned}
& s_{a d}(\phi(x)-v(x))=0, \\
& s_{a d}(\lambda(x) C(x))=0, \\
& s_{a d}(\dot{\lambda}(x)-2 \phi(x))=0, \\
& s_{a d}\left[\lambda(x)\left\{\dot{\phi}(x)-\dot{v}(x)+v^{\prime}(x)-\phi^{\prime}(x)\right\}\right. \\
& \quad+2 i \bar{C}(x) C(x)]=0, \\
& s_{a d} C(x)=0, \\
& s_{a d}\left[\phi(x)\left\{\dot{\phi}(x)-\dot{v}(x)+v^{\prime}(x)-\phi^{\prime}(x)\right\}\right. \\
& \quad+i \bar{C}(x) \dot{C}(x)]=0 .
\end{aligned}
$$

These anti-co-BRST invariant quantities have to be generalized onto the $(2,1)$-dimensional chiral supersubmanifold and the following anti-co-BRST invariant restrictions have to be imposed on the chiral superfields, namely;

$$
\begin{aligned}
& F^{(A D)}(x, \theta)=C(x), \\
& \widetilde{\Phi}(x, \theta)-\widetilde{V}(x, \theta)=\phi(x)-v(x), \\
& \Lambda(x, \theta) F^{(A D)}(x, \theta)=\lambda(x) C(x), \\
& \widetilde{\Phi}(x, \theta) \dot{F}^{(A D)}(x, \theta)=\phi(x) \dot{C}(x), \\
& \dot{\Lambda}(x, \theta)-2 \widetilde{\Phi}(x, \theta)=\dot{\lambda}(x)-2 \phi(x), \\
& \widetilde{\Phi}(x, \theta)\left[\dot{\widetilde{\Phi}}(x, \theta)-\dot{\widetilde{V}}(x, \theta)+\widetilde{V}^{\prime}(x, \theta)-\widetilde{\Phi}^{\prime}(x, \theta)\right] \\
& \quad+i \bar{F}(x, \theta) \dot{F}^{(A D)}(x, \theta)=\phi(x) \\
& \quad \cdot\left[\dot{\phi}(x)-\dot{v}(x)+v^{\prime}(x)-\phi^{\prime}(x)\right]+i \bar{C}(x) \dot{C}(x),
\end{aligned}
$$

$$
\begin{aligned}
& \Lambda(x, \theta)\left[\dot{\widetilde{\Phi}}(x, \theta)-\dot{\vec{V}}(x, \theta)+\widetilde{V}^{\prime}(x, \theta)-\widetilde{\Phi}^{\prime}(x, \theta)\right] \\
& \quad+2 i \bar{F}(x, \theta) F^{(A D)}(x, \theta)=\lambda(x) \\
& \cdot\left[\dot{\phi}(x)-\dot{v}(x)+v^{\prime}(x)-\phi^{\prime}(x)\right]+2 i \bar{C}(x) C(x),
\end{aligned}
$$

where $F^{(A D)}(x, \theta)=C(x)$ is due to the anti-co-BRST invariance $s_{a d} C(x)=0$ of the ghost field $C(x)$. The superscript $(A D)$ on superfield $F(x, \theta)$ denotes that this superfield has been obtained after the anti-co-BRST invariant restriction. The above restrictions (28) lead to the following relationships between the secondary fields and the basic fields of the theory described by the Lagrangian density (1):

$$
\begin{aligned}
& \bar{b}_{1}(x)=0, \\
& \bar{f}_{1}(x)=\bar{f}_{2}=\bar{f}(x), \\
& \bar{R}(x) C(x)=0, \\
& \dot{\bar{R}}(x)-2 i \bar{f}(x)=0, \\
& i \bar{f}(x) \dot{C}(x)=0, \\
& i \bar{f}(x)\left[\dot{\phi}-\dot{v}+v^{\prime}-\phi^{\prime}\right]-\dot{C}(x) \bar{b}_{2}(x)=0, \\
& \bar{R}(x)\left[\dot{\phi}(x)-\dot{v}(x)+v^{\prime}(x)-\phi(x)^{\prime}\right]-2 C(x) \bar{b}_{2}(x) \\
& \quad=0 .
\end{aligned}
$$

A simple choice of $\bar{R}(x)=C(x)$ immediately implies the following expressions for the secondary fields in terms of the basic fields of our theory:

$$
\begin{aligned}
\bar{f}(x) & =-\frac{i}{2} \dot{C}(x) \\
\bar{b}_{2}(x) & =\frac{1}{2}\left[\dot{\phi}(x)-\dot{v}(x)+v^{\prime}(x)+\phi^{\prime}(x)\right] .
\end{aligned}
$$

Thus, it is evident that the anti-co-BRST invariant restrictions (28) lead to the exact determination of the secondary fields in terms of the basic fields.

The substitution of the above values of the secondary fields into the chiral expansions (15) leads to the following uniform expansions:

$$
\begin{gathered}
\widetilde{\Phi}^{(A D)}(x, \theta)=\phi(x)+\theta\left(\frac{\dot{C}(x)}{2}\right) \\
\equiv \phi(x)+\theta\left(s_{a d} \phi(x)\right), \\
\widetilde{V}^{(A D)}(x, \theta)=v(x)+\theta\left(\frac{\dot{C}(x)}{2}\right) \\
\equiv v(x)+\theta\left(s_{a d} v(x)\right),
\end{gathered}
$$




$$
\begin{aligned}
& \Lambda^{(A D)}(x, \theta)=\lambda(x)+\theta(C(x)) \\
& \quad \equiv \lambda(x)+\theta\left(s_{a d} \lambda(x)\right), \\
& F^{(A D)}(x, \theta)=C(x)+\theta(0) \equiv C(x)+\theta\left(s_{a d} C(x)\right), \\
& \bar{F}^{(A D)}(x, \theta) \\
& \quad=\bar{C}(x)+\theta\left[\frac{i}{2}\left\{\dot{\phi}(x)-\dot{v}(x)+v^{\prime}(x)-\phi^{\prime}(x)\right\}\right] \\
& \quad \equiv \bar{C}(x)+\theta\left(s_{a d} \bar{C}(x)\right) .
\end{aligned}
$$

The superscript $(A D)$ on the superfields denotes that we have obtained the above expansions after the application of the anti-dual-BRST (anti-co-BRST) invariant restrictions on the chiral superfields. A close look and careful observation of the expansions (26) and (31) reveals a relationship between the (anti-)co-BRST symmetry transformation $\left(s_{(a) d}\right)$ and the translational generators $\left(\partial_{\theta}\right.$ and $\left.\partial_{\bar{\theta}}\right)$ along the Grassmannian directions of the chiral and antichiral supersubmanifolds of the general $(2,2)$-dimensional supermanifold. The geometrical interpretations of $s_{(a) d}$ are similar to the (anti-)BRST symmetry transformations $s_{(a) b}$ which have already been discussed in our previous section [cf. (21) and discussions after it]. The nilpotency $\left(s_{(a) d}^{2}=0\right)$ of $s_{(a) d}$ is linked with the nilpotency $\left(\partial_{\theta}^{2}=0\right)$ of $\partial_{\theta}$.

\section{Invariance of the Lagrangian Density: Superfield Approach and Geometrical Meaning}

We provide the geometrical interpretation for the BRST invariance of the Lagrangian density (1) within the framework of our augmented superfield formalism. Towards this goal in mind, we generalize the Lagrangian density (1) (defined on the flat $2 D$ ordinary Minkowskian spacetime manifold) onto the $(2,1)$-dimensional antichiral supersubmanifold (of the general $(2,2)$-dimensional supermanifold) as

$$
\begin{aligned}
& \mathscr{L}_{b} \longrightarrow \widetilde{\mathscr{L}}_{b}^{(\mathscr{B})}=\frac{1}{2}\left[\dot{\Phi}^{(\mathscr{B})}(x, \bar{\theta}) \dot{\bar{\Phi}}^{(\mathscr{B})}(x, \bar{\theta})\right. \\
& \left.-\dot{\bar{V}}^{(\mathscr{B})}(x, \bar{\theta}) \dot{\bar{V}}^{(\mathscr{B})}(x, \bar{\theta})\right]+\dot{\bar{V}}^{(\mathscr{B})}(x, \bar{\theta}) \\
& \cdot\left[\widetilde{V}^{\prime(\mathscr{B})}(x, \bar{\theta})-\widetilde{\Phi}^{\prime(\mathscr{B})}(x, \bar{\theta})\right]-\frac{1}{2}\left[\widetilde{\Phi}^{\prime(\mathscr{B})}(x, \bar{\theta})\right. \\
& \left.-\widetilde{V}^{(\mathscr{B})}(x, \bar{\theta})\right]^{2}+\Lambda^{(\mathscr{B})}(x, \bar{\theta})\left[\dot{\widetilde{\Phi}}^{(\mathscr{B})}(x, \bar{\theta})\right. \\
& \left.-\dot{\bar{V}}^{(\mathscr{B})}(x, \bar{\theta})+\widetilde{V}^{\prime(\mathscr{B})}(x, \bar{\theta})-\widetilde{\Phi}^{\prime(\mathscr{B})}(x, \bar{\theta})\right] \\
& +B(x)\left[\dot{\Lambda}^{(\mathscr{B})}(x, \bar{\theta})-\widetilde{V}^{(\mathscr{B})}(x, \bar{\theta})-\widetilde{\Phi}^{(\mathscr{B})}(x, \bar{\theta})\right]
\end{aligned}
$$

$$
\begin{aligned}
& +\frac{B^{2}(x)}{2}-i \dot{\bar{F}}^{(\mathscr{B})}(x, \bar{\theta}) \dot{F}^{(\mathscr{B})}(x, \bar{\theta})+2 i \bar{F}^{(\mathscr{B})}(x, \bar{\theta}) \\
& \cdot F^{(\mathscr{B})}(x, \bar{\theta}),
\end{aligned}
$$

where the superfields are defined on the antichiral supersubmanifold and it would be noted that $F^{(\mathscr{B})}(x, \bar{\theta})=C(x)$. In the above, the superscript $(\mathscr{B})$ stands for the superfields that have been obtained after the application of BRST invariant restrictions. Furthermore, we have the following equations:

$$
\begin{aligned}
& \widetilde{V}^{(\mathscr{B})}(x, \bar{\theta})-\widetilde{\Phi}^{\prime(\mathscr{B})}(x, \bar{\theta})=v^{\prime}(x)-\phi^{\prime}(x), \\
& \dot{\widetilde{\Phi}}^{(\mathscr{B})}(x, \bar{\theta})-\dot{\bar{V}}^{(\mathscr{B})}(x, \bar{\theta})+\widetilde{V}^{\prime(\mathscr{B})}(x, \bar{\theta}) \\
& -\widetilde{\Phi}^{\prime(\mathscr{B})}(x, \bar{\theta})=\dot{\phi}(x)-\dot{v}(x)+v^{\prime}(x)-\phi^{\prime}(x) .
\end{aligned}
$$

In the generalization, $\mathscr{L}_{b} \rightarrow \widetilde{\mathscr{L}}_{b}^{(\mathscr{B})}$, we have taken the expansion from (14). As a consequence of our earlier result in (21), it can be checked that the following is true, namely;

$$
\frac{\partial}{\partial \bar{\theta}} \widetilde{\mathscr{L}}_{b}^{(\mathscr{B})}=\frac{d}{d t}[B \dot{C}] \equiv s_{b} \mathscr{L}_{b}, \quad\left(\partial_{\bar{\theta}} \longleftrightarrow s_{b}\right) .
$$

This relationship provides the geometrical interpretation for the BRST invariance. It states that this super-Lagrangian density $\widetilde{\mathscr{L}}_{b}^{(\mathscr{B})}$ is the sum of composite (super)fields that have been obtained after the application of the BRST invariant restriction (11). This sum is such that its translation along the $\bar{\theta}$-direction of the antichiral supersubmanifold generates a total "time" derivative in the ordinary space [(cf. (3), (34)].

Against the backdrop of the discussion about the BRST invariance, we can also talk about the anti-BRST invariance of the Lagrangian density (1). In this connection, we generalize it on the chiral supersubmanifold [with $\bar{F}^{(A B)}(x, \theta)=\bar{C}(x)$ ] as

$$
\begin{aligned}
& \mathscr{L}_{b} \longrightarrow \widetilde{\mathscr{L}}_{b}^{(A B)}=\frac{1}{2}\left[\dot{\widetilde{\Phi}}^{(A B)}(x, \theta) \dot{\bar{\Phi}}^{(A B)}(x, \theta)\right. \\
& \left.-\dot{\vec{V}}^{(A B)}(x, \theta) \dot{\vec{V}}^{(A B)}(x, \theta)\right]+\dot{\widetilde{V}}^{(A B)}(x, \theta) \\
& \cdot\left[\widetilde{V}^{\prime(A B)}(x, \theta)-\widetilde{\Phi}^{\prime(A B)}(x, \theta)\right]-\frac{1}{2}\left[\widetilde{\Phi}^{\prime(A B)}(x, \theta)\right. \\
& \left.-\widetilde{V}^{(A B)}(x, \theta)\right]^{2}+\Lambda^{(A B)}(x, \theta)\left[\dot{\bar{\Phi}}^{(A B)}(x, \theta)\right. \\
& \left.-\dot{\vec{V}}^{(A B)}(x, \theta)+\widetilde{V}^{\prime(A B)}(x, \theta)-\widetilde{\Phi}^{\prime(A B)}(x, \theta)\right] \\
& +B(x)\left[\dot{\Lambda}^{(A B)}(x, \theta)-\widetilde{V}^{(A B)}(x, \theta)-\widetilde{\Phi}^{(A B)}(x, \theta)\right] \\
& +\frac{B^{2}(x)}{2}-i \dot{\bar{F}}^{(A B)}(x, \theta) \dot{F}^{(A B)}(x, \theta) \\
& +2 i \bar{F}^{(A B)}(x, \theta) F^{(A B)}(x, \theta) \text {, }
\end{aligned}
$$


where the superscript $(A B)$ on the superfields stands for the superfields that have been obtained after the application of anti-BRST invariant restrictions [cf. (20)]. It is straightforward to check that the following is true:

$$
\begin{aligned}
\frac{\partial}{\partial \theta} \widetilde{\mathscr{L}}_{b}^{(\mathscr{B})} & =\frac{d}{d t}(B \dot{\bar{C}}) \equiv s_{a b} \mathscr{L}_{b} \Longleftrightarrow \\
s_{a b} & \longleftrightarrow \partial_{\theta} .
\end{aligned}
$$

Thus, we note that the anti-BRST invariance of the Lagrangian density (1) is captured by the 1.h.s. of the above equation where a derivative $\left(\partial_{\theta}\right)$, acting on the super Lagrangian density $\widetilde{\mathscr{L}}_{b}^{(\mathscr{B})}$ defined on the $(2,1)$-dimensional supersubmanifold, produces the total "time" derivative in the ordinary space (thereby rendering the action integral $S=$ $\int d^{2} x \mathscr{L}_{b} \equiv \int d x \int d t \mathscr{L}_{b}$ invariant). Geometrically, this statement is equivalent to the translation of the super Lagrangian density (35) along the $\theta$-direction of the chiral $(2,1)$ dimensional supersubmanifold (of the general $(2,2)$-dimensional supermanifold) such that this process of translation generates an ordinary total "time" derivative in the ordinary space.

We now focus on the (anti-)co-BRST invariance of the Lagrangian density $\mathscr{L}_{b}$ within the framework of augmented superfield formalism. We note that the starting Lagrangian density (1) can be generalized onto the $(2,1)$-dimensional (anti)chiral supersubmanifolds (of the general $(2,2)$-dimensional supermanifold) as

$$
\begin{aligned}
& \mathscr{L}_{b} \longrightarrow \widetilde{\mathscr{L}}_{b}^{(D)}=\frac{1}{2}\left[\dot{\widetilde{\Phi}}^{(D)}(x, \bar{\theta}) \dot{\widetilde{\Phi}}^{(D)}(x, \bar{\theta})\right. \\
& \left.-\dot{\vec{V}}^{(D)}(x, \bar{\theta}) \dot{\vec{V}}^{(D)}(x, \bar{\theta})\right]+\dot{\vec{V}}^{(D)}(x, \bar{\theta}) \\
& \cdot\left[\widetilde{V}^{\prime(D)}(x, \bar{\theta})-\widetilde{\Phi}^{\prime(D)}(x, \bar{\theta})\right]-\frac{1}{2}\left[\widetilde{\Phi}^{\prime(D)}(x, \bar{\theta})\right. \\
& \left.-\widetilde{V}^{(D)}(x, \bar{\theta})\right]^{2}+\Lambda^{(D)}(x, \bar{\theta})\left[\dot{\Phi}^{(D)}(x, \bar{\theta})\right. \\
& \left.-\dot{\vec{V}}^{(D)}(x, \bar{\theta})+\widetilde{V}^{\prime(D)}(x, \bar{\theta})-\widetilde{\Phi}^{\prime(D)}(x, \bar{\theta})\right] \\
& +B(x)\left[\dot{\Lambda}^{(D)}(x, \bar{\theta})-\widetilde{V}^{(D)}(x, \bar{\theta})-\widetilde{\Phi}^{(D)}(x, \bar{\theta})\right] \\
& +\frac{B^{2}(x)}{2}-i \dot{\bar{F}}^{(D)}(x, \bar{\theta}) \dot{F}^{(D)}(x, \bar{\theta})+2 i \bar{F}^{(D)}(x, \bar{\theta}) \\
& \cdot F^{(D)}(x, \bar{\theta}) \\
& \mathscr{L}_{b} \longrightarrow \widetilde{\mathscr{L}}_{b}^{(A D)}=\frac{1}{2}\left[\dot{\widetilde{\Phi}}^{(A D)}(x, \theta) \dot{\widetilde{\Phi}}^{(A D)}(x, \theta)\right. \\
& \left.-\dot{\vec{V}}^{(A D)}(x, \theta) \dot{\vec{V}}^{(A D)}(x, \theta)\right]+\dot{\vec{V}}^{(A D)}(x, \theta) \\
& \cdot\left[\widetilde{V}^{\prime(A D)}(x, \theta)-\widetilde{\Phi}^{\prime(A D)}(x, \theta)\right]-\frac{1}{2}\left[\widetilde{\Phi}^{\prime(A D)}(x, \theta)\right. \\
& \left.-\widetilde{V}^{(A D)}(x, \theta)\right]^{2}+\Lambda^{(A D)}(x, \theta)\left[\dot{\widetilde{\Phi}}^{(A D)}(x, \theta)\right.
\end{aligned}
$$

$$
\begin{aligned}
& \left.-\dot{\vec{V}}^{(A D)}(x, \theta)+\widetilde{V}^{\prime(A D)}(x, \theta)-\widetilde{\Phi}^{\prime(A D)}(x, \theta)\right] \\
& +B(x)\left[\dot{\Lambda}^{(A D)}(x, \theta)-\widetilde{V}^{(A D)}(x, \theta)-\widetilde{\Phi}^{(A D)}(x, \theta)\right] \\
& +\frac{B^{2}(x)}{2}-i \dot{\bar{F}}^{(A D)}(x, \theta) \dot{F}^{(A D)}(x, \theta) \\
& +2 i \bar{F}^{(A D)}(x, \theta) F^{(A D)}(x, \theta),
\end{aligned}
$$

where the superscripts $(D)$ and $(A D)$ on the superfields denote the superfields that have been obtained after the application of the co-BRST and anti-co-BRST invariant restrictions. It is self-evident that $\bar{F}^{(D)}(x, \bar{\theta})=\bar{C}(x)$ and $F^{(A D)}(x, \theta)=C(x)$ because $s_{d} \bar{C}(x)=0$ and $s_{a d} C(x)=0$. The (anti-)co-BRST invariance [cf. (7)] of the $2 D$ ordinary Lagrangian density (1) can be captured in the language of the augmented superfield formalism (in terms of the operation of the translational generators) as follows:

$$
\begin{gathered}
\frac{\partial}{\partial \theta} \widetilde{\mathscr{L}}_{b}^{(A D)}=\frac{\partial}{\partial t}\left[\frac{\dot{C}}{2}\left(\dot{\phi}-\dot{v}+v^{\prime}-\phi^{\prime}\right)\right] \equiv s_{a d} \mathscr{L}_{b}, \\
\frac{\partial}{\partial \bar{\theta}} \widetilde{\mathscr{L}}_{b}^{(D)}=\frac{\partial}{\partial t}\left[\frac{\dot{\bar{C}}}{2}\left(\dot{\phi}-\dot{v}+v^{\prime}-\phi^{\prime}\right)\right] \equiv s_{d} \mathscr{L}_{b} .
\end{gathered}
$$

Thus, we find that $\partial_{\theta} \leftrightarrow s_{a d}$ and $\partial_{\bar{\theta}} \leftrightarrow s_{d}$. In other words, we note that the (anti-)co-BRST invariance of the Lagrangian density $\mathscr{L}_{b}$ [cf. (7)] can be captured within the framework of augmented superfield formalism as the translations of the super-Lagrangian densities $\mathscr{L}_{b}^{((A) D)}$ along the $\theta$ and $\bar{\theta}$ directions of the chiral and antichiral super-submanifolds such that these translations generate the ordinary "time" derivatives in the $2 D$ ordinary Minkowskian space thereby rendering the action integral $S=\int d^{2} x \mathscr{L}_{b}$ invariant (for the physically well-defined fields of our theory which vanish off at infinity).

We end this section reemphasizing the fact that the (anti-)BRST and (anti-)co-BRST invariance of the Lagrangian density (1) in the ordinary $2 D$ flat spacetime manifold is equivalent to the translation of the corresponding superLagrangian densities [cf. (32), (35), and (37)] along the $(\bar{\theta}) \theta$ directions of the (anti)chiral supersubmanifolds (of the general $(2,2)$-dimensional supermanifold) such that this process of translation on these supermanifolds generates the ordinary "time" derivatives. This is the geometrical interpretation for the (anti-)BRST and (anti-)co-BRST invariances that are present in our theory.

\section{Nilpotency and Absolute Anticommutativity Properties: Superfield Formalism}

We capture here the nilpotency and absolute anticommutativity properties of the conserved (anti-)BRST and (anti-)coBRST charges which are the generators for such symmetry transformations (that have been christened as (anti-)BRST 
and (anti-)co-BRST symmetry transformations). It can be readily checked that the following are true, namely;

$$
\begin{aligned}
Q_{b} & =\int d x s_{b}[-i(\bar{C} \dot{C}-\dot{\bar{C}} C)] \\
& \equiv \int d x\left[s_{a b}(-i \dot{C} C)\right], \\
Q_{a b} & =\int d x s_{a b}[i(\bar{C} \dot{C}-\dot{\bar{C}} C)] \equiv \int d x\left[s_{b}(i \dot{\bar{C}} \bar{C})\right],
\end{aligned}
$$

where the (anti-)BRST transformations $\left(s_{(a) b}\right)$ are given in (2) and the expressions for the (anti-)BRST charges are quoted in (4). In view of the mappings, $s_{b} \leftrightarrow \partial_{\bar{\theta}}, s_{a b} \leftrightarrow \partial_{\theta}$, we can express (39) in terms of the superfields (with $F^{(\mathscr{B})}(x, \bar{\theta})=$ $\left.C(x), \bar{F}^{(A B)}(x, \theta)=\bar{C}(x)\right)$ as follows:

$$
\begin{aligned}
Q_{b} & =-i \int d x\left[\frac{\partial}{\partial \bar{\theta}}\left(\bar{F}^{(\mathscr{B})}(x, \bar{\theta}) \dot{F}^{(\mathscr{B})}(x, \bar{\theta})-\dot{\bar{F}}^{(\mathscr{B})}(x, \bar{\theta}) F^{(\mathscr{B})}(x, \bar{\theta})\right)\right] \\
& \equiv-i \int d x\left[\int d \bar{\theta}\left(\bar{F}^{(\mathscr{B})}(x, \bar{\theta}) \dot{F}^{(\mathscr{B})}(x, \bar{\theta})-\dot{\bar{F}}^{(\mathscr{B})}(x, \bar{\theta}) F^{(\mathscr{B})}(x, \bar{\theta})\right)\right], \\
Q_{b} & =-i \int d x\left[\frac{\partial}{\partial \theta}\left(\dot{F}^{(A B)}(x, \theta) F^{(A B)}(x, \theta)\right)\right] \equiv-i \int d x\left[\int d \theta\left(\dot{F}^{(A B)}(x, \theta) F^{(A B)}(x, \theta)\right)\right], \\
Q_{a b} & =i \int d x\left[\frac{\partial}{\partial \theta}\left(\bar{F}^{(A B)}(x, \theta) \dot{F}^{(A B)}(x, \theta)-\dot{\bar{F}}^{(A B)}(x, \theta) F^{(A B)}(x, \theta)\right)\right] \\
& \equiv i \int d x\left[\int d \theta\left(\bar{F}^{(A B)}(x, \theta) \dot{F}^{(A B)}(x, \theta)-\dot{\bar{F}}^{(A B)}(x, \theta) F^{(A B)}(x, \theta)\right)\right], \\
Q_{a b} & =i \int d x\left[\frac{\partial}{\partial \bar{\theta}}\left(\dot{\bar{F}}^{(\mathscr{B})}(x, \bar{\theta}) \bar{F}^{(\mathscr{B})}(x, \bar{\theta})\right)\right] \equiv i \int d x\left[\int d \bar{\theta}\left(\dot{\bar{F}}^{(\mathscr{B})}(x, \bar{\theta}) \bar{F}^{(\mathscr{B})}(x, \bar{\theta})\right)\right] .
\end{aligned}
$$

The above equations immediately imply that $\partial_{\bar{\theta}} Q_{b}=0$ due to the nilpotency $\left(\partial_{\bar{\theta}}^{2}=0\right)$ of $\partial_{\bar{\theta}}$. In exactly similar fashion, we note that $\partial_{\theta} Q_{a b}=0$ due to the nilpotency $\left(\partial_{\theta}^{2}=0\right)$ of the translational generator $\partial_{\theta}$. However, when we translate the above observations in the language of ordinary (anti-)co-BRST symmetry transformations and charges, we have

$$
\begin{aligned}
\partial_{\bar{\theta}} Q_{b} & =0 \Longleftrightarrow \\
s_{b} Q_{b} & =i\left\{Q_{b}, Q_{b}\right\}=0 \Longrightarrow \\
Q_{b}^{2} & =0, \\
\partial_{\theta} Q_{a b} & =0 \Longleftrightarrow \\
s_{a b} Q_{a b} & =i\left\{Q_{a b}, Q_{a b}\right\}=0 \Longrightarrow \\
Q_{a b}^{2} & =0 .
\end{aligned}
$$

Thus, we observe that the nilpotency $\left(Q_{(a) b}^{2}=0\right)$ of the (anti-)BRST charges $Q_{(a) b}$ is connected with the nilpotency $\left(\partial_{\theta}^{2}=\partial_{\bar{\theta}}^{2}=0\right)$ of the translational generators $\left(\partial_{\theta}, \partial_{\bar{\theta}}\right)$ along the (anti-)chiral supersubmanifolds of the general $(2,2)$ dimensional supermanifold.
It is very interesting to observe that $\partial_{\theta} Q_{b}=0$ and $\partial_{\bar{\theta}} Q_{a b}=$ 0 , too. This can be translated into the ordinary $2 D$ space (with $\left.s_{b} \leftrightarrow \partial_{\bar{\theta}}, s_{a b} \leftrightarrow \partial_{\theta}\right)$ as follows:

$$
\begin{aligned}
\partial_{\theta} Q_{b} & =0 \Longleftrightarrow \\
s_{a b} Q_{b} & =i\left\{Q_{b}, Q_{a b}\right\}=0 \Longrightarrow \\
Q_{b} Q_{a b}+Q_{a b} Q_{b} & =0, \\
\partial_{\bar{\theta}} Q_{a b} & =0 \Longleftrightarrow \\
s_{b} Q_{a b} & =i\left\{Q_{a b}, Q_{b}\right\}=0 \Longrightarrow \\
Q_{a b} Q_{b}+Q_{b} Q_{a b} & =0 .
\end{aligned}
$$

Thus, the nilpotency and absolute anticommutativity of the (anti-)BRST charges can be captured in the language of augmented superfield formalism. In other words, the nilpotency $\left(\partial_{\theta}^{2}=\partial_{\bar{\theta}}^{2}=0\right)$ of the translational generators as well as absolute anticommutativity $\left(\partial_{\theta} \partial_{\bar{\theta}}+\partial_{\bar{\theta}} \partial_{\theta}=0\right)$ of the translational generators $\left(\partial_{\theta}, \partial_{\bar{\theta}}\right)$ on the (anti)chiral supersubmanifolds is intimately connected with such properties associated with the (anti-)BRST charges $Q_{(a) b}$ (and the (anti-)BRST symmetry transformations $s_{(a) b}$ they generate). The symmetries $s_{(a) b}$ also satisfy $s_{(a) b}^{2}=0$ as well as $s_{b} s_{a b}+s_{a b} s_{b}=0$ which are nothing but the nilpotency and absolute anticommutativity properties of the (anti-)BRST symmetry transformations. 
We now concentrate on the nilpotency and absolute anticommutativity properties of the (anti-)co-BRST charges $Q_{(a) d}$ and the symmetry translations they generate. It can be checked that the conserved (anti-)co-BRST charges can be expressed as

$$
\begin{aligned}
Q_{d} & =i \int d x s_{d}[C \dot{\bar{C}}+\bar{C} \dot{C}] \equiv i \int d x s_{a d}(\dot{\bar{C}} \bar{C}), \\
Q_{a d} & =i \int d x s_{a d}[\dot{\bar{C}}+\bar{C} \dot{C}] \equiv-i \int d x s_{d}(\dot{C} C) .
\end{aligned}
$$

The above expressions for the (anti-)co-BRST charges $Q_{(a) d}$ can be also expressed in terms of the superfields, Grassmannian differentials, and derivatives in view of the general relationship: $s_{d} \leftrightarrow \partial_{\bar{\theta}}, s_{a d} \leftrightarrow \partial_{\theta}$. In other words, we have the following expressions for the (anti-)co-BRST charges $Q_{(a) d}$ within the framework of augmented superfield formalism:

$$
\begin{aligned}
Q_{d} & =\int d x\left[\frac{\partial}{\partial \bar{\theta}}\left(i \bar{F}^{(D)}(x, \bar{\theta}) \dot{F}^{(D)}(x, \bar{\theta})-i \dot{\bar{F}}^{(D)}(x, \bar{\theta}) F^{(D)}(x, \bar{\theta})\right)\right] \\
& \equiv \int d x\left[\int d \bar{\theta}\left(i \bar{F}^{(D)}(x, \bar{\theta}) \dot{F}^{(D)}(x, \bar{\theta})-i \dot{\bar{F}}^{(D)}(x, \bar{\theta}) F^{(D)}(x, \bar{\theta})\right)\right], \\
Q_{d} & =\int d x\left[\frac{\partial}{\partial \theta}\left(i \dot{\bar{F}}^{(A D)}(x, \theta) \bar{F}^{(A D)}(x, \theta)\right)\right] \equiv \int d x\left[\int d \theta\left(\dot{\bar{F}}^{(A D)}(x, \theta) \bar{F}^{(A D)}(x, \theta)\right)\right], \\
Q_{a d} & =\int d x\left[\frac{\partial}{\partial \theta}\left(i \bar{F}^{(A D)}(x, \theta) \dot{F}^{(A D)}(x, \theta)-i \dot{\bar{F}}^{(A D)}(x, \theta)\right) F^{(A D)}(x, \theta)\right] \\
& \equiv \int d x\left[\int d \theta\left(i \bar{F}^{(A D)}(x, \theta) \dot{F}^{(A D)}(x, \theta)-i \dot{\bar{F}}^{(A D)}(x, \theta)\right) F^{(A D)}(x, \theta)\right], \\
Q_{a d} & =-i \int d x\left[\frac{\partial}{\partial \bar{\theta}}\left(\dot{F}^{(D)}(x, \bar{\theta}) F^{(D)}(x, \bar{\theta})\right)\right] \equiv-i \int d x\left[\int d \bar{\theta}\left(\dot{F}^{(D)}(x, \bar{\theta}) F^{(D)}(x, \bar{\theta})\right)\right],
\end{aligned}
$$

where, as is evident from our earlier discussions, we have taken $\bar{F}^{(D)}(x, \bar{\theta})=\bar{C}(x), F^{(A D)}(x, \theta)=C(x)$ due to $s_{d} \bar{C}(x)=$ 0 and $s_{a d} C(x)=0$. Thus, we note that, as is the case with the (anti-)BRST charges, there are two types of representations for the conserved (anti-)co-BRST charges $Q_{(a) d}$ in the language of superspace coordinates, superfields, Grassmannian differentials, and derivatives, too.

The nilpotency and absolute anticommutativity of the (ant-)co-BRST charges $Q_{(a) d}$ are hidden in the above expressions for these charges within the framework of superfield formalism. For instance, it can be seen that the following is true, namely;

$$
\begin{aligned}
\partial_{\bar{\theta}} Q_{d} & =0 \Longleftrightarrow \\
s_{d} Q_{d} & =i\left\{Q_{d}, Q_{d}\right\} \Longrightarrow \\
Q_{d}^{2} & =0, \\
\partial_{\theta} Q_{a d} & =0 \Longleftrightarrow \\
s_{a d} Q_{a d} & =i\left\{Q_{a d}, Q_{a d}\right\} \Longrightarrow \\
Q_{a d}^{2} & =0 .
\end{aligned}
$$

The above equations demonstrate that the nilpotency $\left(Q_{(a) d}^{2}=\right.$ $0)$ of $Q_{(a) d}$ and nilpotency of $\left(\partial_{\theta}^{2}=\partial_{\bar{\theta}}^{2}=0\right)$ of the translational generators $\left(\partial_{\theta}, \partial_{\bar{\theta}}\right)$ are interconnected. Furthermore, we note that the alternative expressions for $Q_{(a) d}$, in the above equations, are such that

$$
\begin{aligned}
\partial_{\theta} Q_{d} & =0 \Longleftrightarrow \\
s_{a d} Q_{d} & =i\left\{Q_{d}, Q_{a d}\right\}=0 \Longrightarrow \\
Q_{d} Q_{a d}+Q_{a d} Q_{d} & =0, \\
\partial_{\bar{\theta}} Q_{a d} & =0 \Longleftrightarrow \\
s_{d} Q_{a d} & =i\left\{Q_{a d}, Q_{d}\right\}=0 \Longrightarrow \\
Q_{a d} Q_{d}+Q_{d} Q_{a d} & =0,
\end{aligned}
$$

which demonstrate that the nilpotency $\left(\partial_{\theta}^{2}=\partial_{\bar{\theta}}^{2}=0\right)$ of the translational generators $\left(\partial_{\theta}\right.$ and $\left.\partial_{\bar{\theta}}\right)$ is also connected with the absolute anticommutativity $\left(\partial_{\theta} \partial_{\bar{\theta}}+\partial_{\bar{\theta}} \partial_{\theta}=0\right)$ properties. This is due to the fact that the nilpotency property $\left(\partial_{\theta}^{2}=\partial_{\bar{\theta}}^{2}=0\right)$ is the limiting case of the absolute anticommutativity $\left(\partial_{\theta} \partial_{\bar{\theta}}+\right.$ $\partial_{\bar{\theta}} \partial_{\theta}=0$ ) when $\partial_{\theta}=\partial_{\bar{\theta}}\left(\right.$ or $\partial_{\bar{\theta}}=\partial_{\theta}$ ). This is the reason that we see that, in the ordinary $2 D$ system, we have the validity of relations: $Q_{(a) d}^{2}=0$ and $Q_{a d} Q_{d}+Q_{d} Q_{a d}=0$. We conclude that the nilpotency and absolute anticommutativity properties of the (anti-)co-BRST charges (and the continuous symmetries $s_{(a) d}$ they generate) are intimately connected with such properties associated with the translational generators $\left(\partial_{\theta}, \partial_{\bar{\theta}}\right)$ 
along the Grassmannian directions of the $(2,1)$-dimensional chiral and antichiral supersubmanifolds of the general $(2,2)$ dimensional supermanifold (on which our present $2 D$ selfdual chiral bosonic field theory is generalized).

\section{Conclusions}

The central theme of our present investigation has been to provide theoretical richness in the realm of superfield approach to BRST formalism. We have exploited the potential and power of the symmetry invariant restrictions on the (super)fields to derive the nilpotent and absolutely anticommuting (anti-)BRST and (anti-)co-BRST symmetries. We have also provided the geometrical basis for the symmetry transformations, nilpotency, absolute anticommutativity, and symmetry invariance of the Lagrangian density of the $2 D$ self-dual chiral bosonic field theory (within the framework of augmented superfield formalism).

One of the key results of our present investigation has been the observation that we do not need the mathematical strength of the (dual-)horizontality conditions to derive the proper (anti-)BRST and (anti-)co-BRST symmetry transformations. Further, we have found that the geometrical interpretation for the symmetry transformations remains intact in our present method of derivation vis-à-vis the ones where the mathematical strength of the (dual-)horizontality conditions is properly utilized [37]. Thus, both methods of derivation are consistent and, in some cases, complementary to each other (see, e.g., $[20,21,33,34]$ ).

The other key result of our present endeavor is the observation that the nilpotency $\left(Q_{(a) b}^{2}=0, Q_{(a) d}^{2}=0\right)$ and absolute anticommutativity $\left(Q_{b} Q_{a b}+Q_{a b} Q_{b}=0, Q_{d} Q_{a d}+Q_{a d} Q_{d}=0\right)$ of the (anti-)BRST and (anti-)co-BRST charges $\left(Q_{(a) b}\right.$ and $\left.Q_{(a) d}\right)$ are captured within our present method of superfield formalism where we have considered only the (anti)chiral superfields for our whole discussion. This is a novel result in our previous $[33,34]$ and present investigations. These two sacrosanct properties emerge automatically in the superfield approach where the full expansions of superfields along all the Grassmannian directions $(1, \theta, \bar{\theta}, \theta \bar{\theta})$ of the supermanifolds are taken into account (see, e.g., $[20,21]$ ).

One of the shortcomings of our present method of superfield formalism is the fact that it is valid only for those systems where the Curci-Ferrari (CF) conditions [38] turn out to be trivial so that there exists a single (anti-)BRST and (anti-)coBRST invariant Lagrangian density for the system and nilpotency and absolute anticommutativity of the (anti-)BRST and (anti-)co-BRST symmetry transformations are satisfied in a straightforward manner. The $2 D$ self-dual chiral bosonic field theory (which is the topic of discussion for our present endeavor) belongs to this category. Hence, our method is applicable (as the CF-type condition is trivial here). In the cases where the CF conditions are nontrivial, it is the full expansions of the superfields that are applicable because the CF-type conditions as well as the derivation of the proper (anti-)BRST and (anti-)co-BRST symmetries result in very naturally in such kind of superfield formalism. We emphasize that, in the latter case, the (dual-)horizontality conditions are necessarily exploited, too.
It would be a nice future endeavor for us to check the sanctity of our theoretical method in the context of other physical systems of interest. In particular, we are very much eager to apply our method for the BRST description of the SUSY gauge theories which are important because of their relevance (i) in the context of search for the existence of the SUSY particles and (ii) as the field theoretic limiting cases of the (super)string theories. We have successfully applied our method to the description of $\mathcal{N}=2$ SUSY quantum mechanical models [29-32] but the challenge is to apply our method to the description of $\mathcal{N}=2,4,8$ SUSY gauge theories. We are currently busy with these ideas and we plan to report our progress in our future publications.

\section{Competing Interests}

The authors declare that they have no competing interests.

\section{Acknowledgments}

T. Bhanja would like to gratefully acknowledge the financial support from BHU-fellowship under which the present investigation has been carried out.

\section{References}

[1] N. Marcus and J. H. Schwarz, "Field theories that have no manifestly Lorentz-invariant formulation," Physics Letters. B, vol. 115, no. 2, pp. 111-114, 1982.

[2] D. J. Gross, J. A. Harvey, E. Martinec, and R. Rohm, "Heterotic string," Physical Review Letters, vol. 54, no. 6, pp. 502-505, 1985.

[3] X. G. Wen, "Electrodynamical properties of gapless edge excitations in the fractional quantum Hall states," Physical Review Letters, vol. 64, no. 18, pp. 2206-2209, 1990.

[4] M. Stone, "Superfluid dynamics of the fractional quantum Hall state," Physical Review B, vol. 42, no. 1, pp. 212-217, 1990.

[5] J. V. José, L. P. Kadanoff, S. Kirkpatrick, and D. R. Nelson, "Renormalization, vortices, and symmetry-breaking perturbations in the two-dimensional planar model," Physical Review B, vol. 16, no. 3, pp. 1217-1241, 1977.

[6] P. Weigman, "One-dimensional Fermi system and plane xy model," Journal of Physics C: Solid State Physics, vol. 11, no. 8, p. 1583, 1987.

[7] D. Boyanovsky and R. Holman, "The Coulomb gas, vertex operators and the c-theorem," Nuclear Physics B, vol. 332, no. 3, pp. 641-665, 1990.

[8] R. Floreanini and R. Jackiw, "Self-dual fields as charge-density solitons," Physical Review Letters, vol. 59, no. 17, pp. 1873-1876, 1987.

[9] W. Siegel, "Manifest Lorentz invariance sometimes requires non-linearity," Nuclear Physics B, vol. 238, no. 2, pp. 307-316, 1984.

[10] C. Imbimbo and A. Schwimmer, "The lagrangian formulation of chiral scalars," Physics Letters B, vol. 193, no. 4, pp. 455-458, 1987.

[11] J. M. Labastida and M. Pernici, "Heterotic-string Lagrangean in the bosonic formulation," Physical Review Letters, vol. 59, no. 22, pp. 2511-2514, 1987.

[12] P. P. Srivastava, "Quantization of self-dual field revisited," Physical Review Letters, vol. 63, no. 26, pp. 2791-2792, 1989. 
[13] S. Upadhyay and B. P. Mandal, "The model for self-dual chiral bosons as a Hodge theory," European Physical Journal C, vol. 71, no. 9, article 1759, 2011.

[14] C. Becchi, A. Rouet, and R. Stora, "The abelian Higgs Kibble model, unitarity of the S-operator," Physics Letters B, vol. 52, no. 3, pp. 344-346, 1974.

[15] C. Becchi, A. Rouet, and R. Stora, "Renormalization of the abelian Higgs-Kibble model," Communications in Mathematical Physics, vol. 42, pp. 127-162, 1975.

[16] C. Becchi, A. Rouet, and R. Stora, "Renormalization of gauge theories," Annals of Physics, vol. 98, no. 2, pp. 287-321, 1976.

[17] I. V. Tyutin, "Gauge Invariance in field theory and statistical physics in operator formalism," Report FIAN-39, Lebedev Institute, 1975.

[18] J. Thierry-Mieg, "Geometrical reinterpretation of FaddeevPopov ghost particles and BRS transformations," Journal of Mathematical Physics, vol. 21, no. 12, pp. 2834-2838, 1980.

[19] M. Quirós, F. J. de Urries, J. Hoyos, M. L. Mazón, and E. Rodriguez, "Geometrical structure of Faddeev-Popov fields and invariance properties of gauge theories," Journal of Mathematical Physics, vol. 22, no. 8, pp. 1767-1774, 1981.

[20] L. Bonora and M. Tonin, "Superfield formulation of extended BRS symmetry," Physics Letters B, vol. 98, no. 1-2, pp. 48-50, 1981.

[21] L. Bonora, P. Pasti, and M. Tonin, "Geometric description of extended BRS symmetry in superfield formulation," Il Nuovo Cimento A, vol. 63, no. 3, pp. 353-364, 1981.

[22] R. Delbourgo and P. D. Jarvis, "Extended BRS invariance and OSp (4/2) supersymmetry," Journal of Physics A: Mathematical and General, vol. 15, no. 2, pp. 611-625, 1982.

[23] R. Delbourgo, P. D. Jarvis, and G. Thompson, "Local OSp(4/2) supersymmetry and extended BRS transformations for gravity," Physics Letters B, vol. 109, no. 1-2, pp. 25-27, 1982.

[24] L. Alvarez-Gaumé and L. Baulieu, "The two quantum symmetries associated with a classical symmetry," Nuclear Physics B, vol. 212, no. 2, pp. 255-267, 1983.

[25] R. P. Malik and B. P. Mandal, "Augmented superfield approach to exact nilpotent symmetries for matter fields in non-Abelian theory," The European Physical Journal C, vol. 47, no. 1, pp. 219226, 2006.

[26] R. P. Malik, "Augmented superfield approach to unique nilpotent symmetries for complex scalar fields in QED," The European Physical Journal C, vol. 48, no. 3, pp. 825-834, 2006.

[27] R. Malik, "An alternative to the horizontality condition in the superfield approach to BRST symmetries," The European Physical Journal C, vol. 51, no. 1, pp. 169-177, 2007.

[28] P. Malik, "A generalization of the horizontality condition in the superfield approach to nilpotent symmetries for QED with complex scalar fields," Journal of Physics A, vol. 40, no. 18, pp. 4877-4894, 2007.

[29] S. Krishna and R. P. Malik, "N=2 SUSY symmetries for a moving charged particle under influence of a magnetic field: supervariable approach," Annals of Physics, vol. 355, pp. 204216, 2015.

[30] S. Krishna, A. Shukla, and R. P. Malik, "General N=2 supersymmetric quantum mechanical model: supervariable approach to its off-shell nilpotent symmetries," Annals of Physics, vol. 351, pp. 558-570, 2014.

[31] S. Krishna, A. Shukla, and R. P. Malik, "Supervariable approach to nilpotent symmetries of a couple of $N=2$ supersymmetric quantum mechanical models," Canadian Journal of Physics, vol. 92, no. 12, pp. 1623-1631, 2014.
[32] S. Krishna and R. P. Malik, "A free $N=2$ supersymmetric system: novel symmetries," EPL (Europhysics Letters), vol. 109, no. 3, Article ID 31001, 2015.

[33] N. Srinivas, A. Shukla, and R. P. Malik, "Nilpotent symmetries of the 4D Abelian 2-form gauge theory: augmented superfield formalism," https://arxiv.org/abs/1501.06770.

[34] T. Bhanja, N. Srinivas, and R. P. Malik, "(Anti-)BRST and (Anti)co-BRST Symmetries of a System of Rigid Rotor: Augmented Supervariable Approach," https://arxiv.org/abs/1504.04237.

[35] U. Kulshreshtha, D. S. Kulshreshthra, and H. J. W. MueullerKirsten, "A gauge-invariant theory of chiral bosons: WessZumino term, Hamiltonian and BRST formulations," Zeitschrift für Physik C, vol. 60, no. 3, pp. 427-431, 1993.

[36] N. Nakanishi and I. Ojima, Covariant Operator Formalism of Gauge Theory and Quantum Gravity, World Scientific, Singapore, 1990.

[37] D. Shukla, T. Bhanja, and R. P. Malik, "Self-dual chiral boson: augmented superfield approach," The European Physical Journal C, vol. 74, article 3025, 2014.

[38] G. Curci and R. Ferrari, "Slavnov transformations and supersummetry," Physics Letters. B, vol. 63, no. 1, pp. 91-94, 1976. 

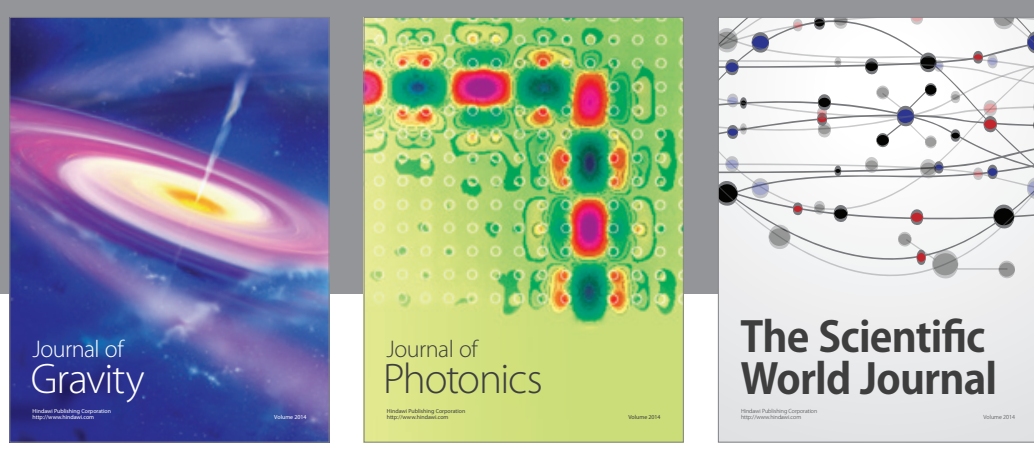

The Scientific World Journal
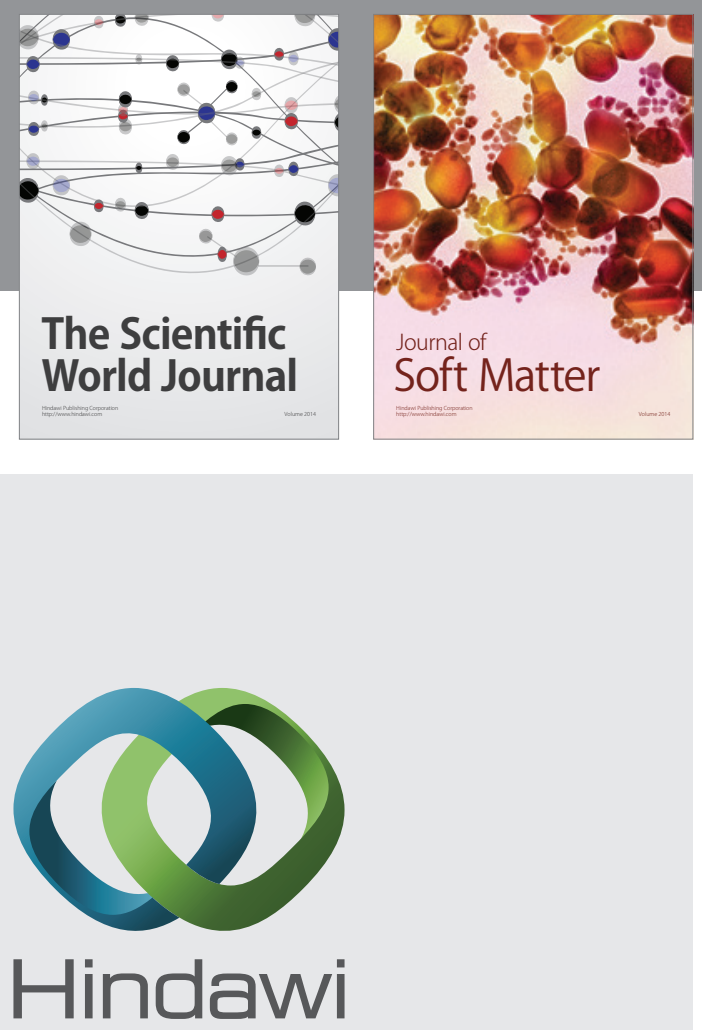

Submit your manuscripts at

https://www.hindawi.com
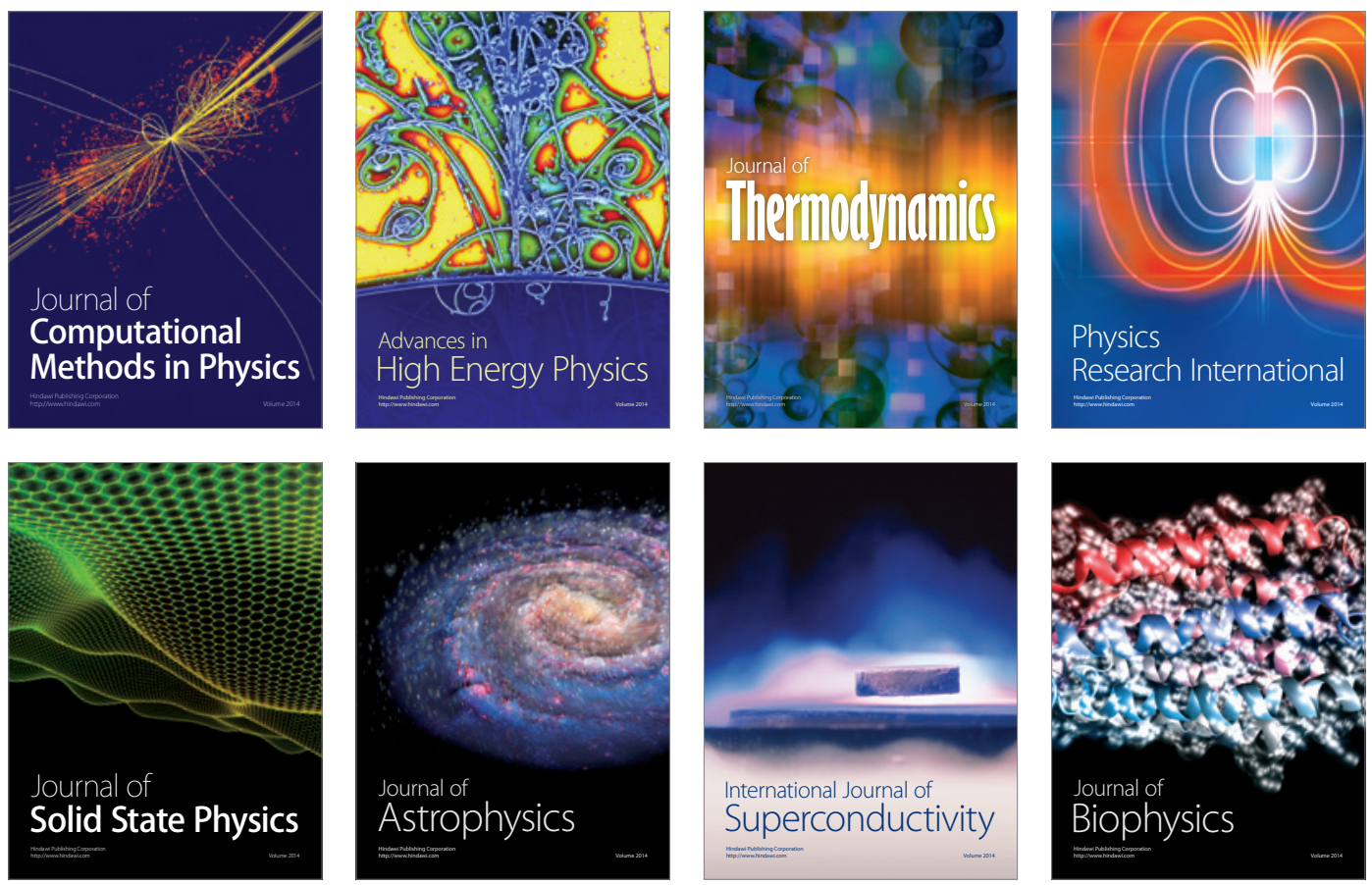
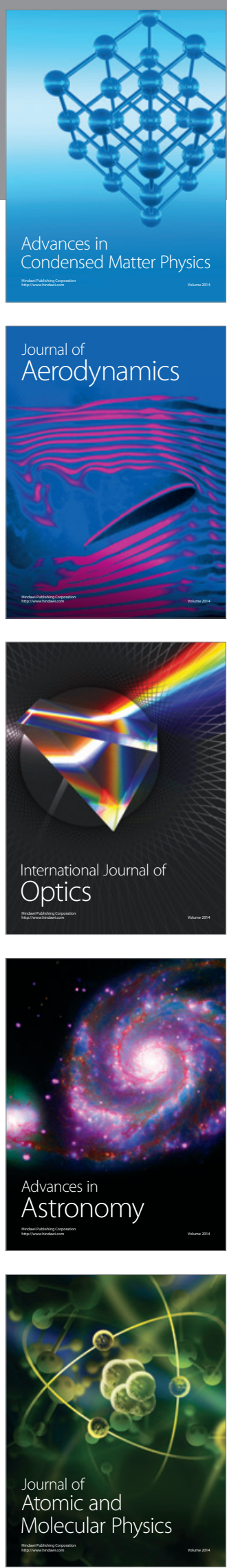